\title{
EVALUATION OF ROLE OF FAST VS CECT ABDOMEN IN DETECTING ORGAN INJURY IN BLUNT ABDOMEN TRAUMA PATIENTS
}

\author{
Balaji Durairaj ${ }^{1}$ Rajesh Chakkarapani²
}

${ }_{1}^{1}$ Assistant Professor, Department of General Surgery, SRM Medical College and Research Institute.

${ }^{2}$ Assistant Professor, Department of General Surgery, SRM Medical College and Research Institute.

\begin{abstract}
BACKGROUND
ABSTRACT

There is rapid increase in the number of blunt abdomen injury patients, mainly due to road traffic accidents. The physical signs are often masked in these patients and usually there is delay in the diagnosis of these patients. The computerised tomographic scan is the investigation of choice in such patients. Focused abdominal sonography in trauma (FAST) also has been proved to be accurate, non-invasive and less time consuming in assessing the critically traumatised patient in the emergency room.

So, this study was undertaken to evaluate the role of FAST/ CECT Abdomen in detecting organ injury in Blunt Abdomen Trauma patients.
\end{abstract}

\section{MATERIALS AND METHODS}

This descriptive study was done among blunt injury abdomen patients who needed laparotomy in SRM Medical College and Research Institute, Potheri. In all patients, FAST and CECT Abdomen was done and compared with preoperative findings.

\section{RESULTS}

A total of 50 cases of blunt injury abdomen were included in this study. Of these cases, 44 cases (88\%) were males and 06 cases $(12 \%)$ were females. The range of age of the patients studied was from 16 years to 72 years and the mean age of the study group was 37.22 years. In our study, Road traffic accidents were the predominant cause of blunt injury. FAST and CECT findings were correlated with per-operative findings. Per-operative findings were positive in 50 patients. 49 patients were correctly identified by FAST (sensitivity 98\%) and CECT correctly identified all 50 patients (sensitivity $100 \%$ ).

\section{CONCLUSION}

CECT Abdomen is found to be highly reliable in detecting blunt abdomen injuries.

\section{KEYWORDS}

FAST, CECT Abdomen, Blunt Abdomen Injuries.

HOW TO CITE THIS ARTICLE: Durairaj B, Chakkarapani R. Evaluation of role of FAST vs CECT abdomen in detecting organ injury in blunt abdomen trauma patients. J. Evolution Med. Dent. Sci. 2018;7(10):1232-1236, DOI: 10.14260/jemds/2018/281

\section{BACKGROUND}

Blunt Abdominal trauma is one of the most common causes among injuries caused mainly due to road traffic accidents. The rapid increase in number of motor vehicles and its aftermath has caused rapid increase in number of victims to blunt abdominal trauma. Motor vehicle accidents account for $75 \%$ to $80 \%$ of blunt abdominal trauma. ${ }^{1}$

Blunt injury of abdomen is also a result of fall from height, assault with blunt objects, industrial mishaps, sport injuries, bomb blasts and fall from riding bicycle. Blunt abdominal trauma is usually not obvious. ${ }^{2}$ Hence, often missed, unless repeatedly looked for. Due to the delay in diagnosis and inadequate treatment of the abdominal injuries, most of the cases are fatal.

Diagnostic CT findings considered for bowel injury are contrast extravasation and/ or extraluminal air. CT diagnosis for small bowel perforation has a sensitivity of $92 \%$ and specificity of $94 \%$. It is often difficult to diagnose the injuries to the retroperitoneum, especially in the presence of other

'Financial or Other Competing Interest': None.

Submission 22-01-2018, Peer Review 14-02-2018,

Acceptance 21-02-2018, Published 05-03-2018.

Corresponding Author:

Dr. Balaji Durairaj,

No. 86, Sadasivam Road,

Kattankulathur-603203, Kanchipuram District.

E-mail: trace.balaji@gmail.com

DOI: $10.14260 /$ jemds $/ 2018 / 281$ injury, as the physical signs may be masked. Intraperitoneal diagnostic tests such as ultrasound and diagnostic peritoneal lavage may be negative. The computerised tomographic scan is the investigation of choice in such patients. ${ }^{3}$

Focused abdominal sonography in trauma (FAST) was familiarised in the United States by Rozycki in the early 1990s. Later studies proved that FAST was accurate, non-invasive and expeditious in assessing the critically traumatised patient in the emergency room. ${ }^{4}$ As quality ultrasound machines have become portable, there is an increasing trend of their application in the initial assessment of blunt abdominal injury. The procedure can be done by surgeons as well as radiologists, both of equal reliability and was particularly useful in detecting haemoperitoneum in the abdominal cavity. In majority cases, FAST can be completed within 3 or 4 minutes. The test is useful for detecting intra-abdominal bleeding in the multiple injured or pregnant patients. ${ }^{5}$

Drawback of FAST is the fact that a positive examination depends on the presence of free intraperitoneal fluid, and is operator dependent. With most operators, ultrasound will detect a minimum of $200 \mathrm{~mL}$ of fluid. Injuries without haemoperitoneum may be not detected by this modality. ${ }^{6}$ In addition, the FAST examination is not used to grade solid organ injuries. Therefore, in the haemodynamically stable patient, a follow-up CECT scan should be done if conservative management is contemplated. FAST compares favourably with most traditionally utilised diagnostic investigations. ${ }^{7}$ 
Computerised tomography of abdomen (CECT scan) provides important diagnostic information on abdominal injuries. CECT has become the 'gold standard' for the intraabdominal diagnosis of injury in a stable patient. The scan is performed using intravenous contrast and often oral contrast as well. CECT also grades solid organ injury and in a stable individual with positive FAST findings. It is indicated to grade organ injury and to evaluate contrast extravasation. If contrast is seen extravasating, even with low-grade hepatic or splenic injuries, exploratory laparotomy or more recently interventional angiography and intravascular embolisation are done. ${ }^{8}$ In view of increasing number of blunt abdomen injury patients, this study has been done to study the sensitivity of FAST in comparison with CECT in blunt injury abdomen patients in a tertiary care hospital in Chennai.

\section{MATERIALS AND METHODS}

This descriptive study was done among blunt injury abdomen patients who needed laparotomy in SRM Medical College and Research Institute, Potheri. The patients with associated chest and head injury were excluded from the study. Methods of collection of data included history taking, clinical examination and relevant diagnostic investigations performed over the patient.

All injured were brought to an emergency resuscitation area where primary-survey, resuscitation and a secondary survey in a standard method was conducted. The FAST investigation was performed during primary-survey, after securing airway and establishing the adequate oxygenation/ventilation patients were scanned by a FAST investigation. The FAST investigation was performed using 4 windows: Subxiphoid, right-upper quadrant (Morrison's space), left upper quadrant and suprapubic. The critical windows for intra-abdominal bleeding were the hepatorenal space (Morrison's space), the splenorenal space and the pelvic pouch of Douglas. The USG machine kept in the trauma ward resuscitation area was used for these examinations.

The FAST investigation was classified as positive (clearly showing fluid on at least one window) or negative (good scan in at least three views, no fluid). No quantitative score system was used for the quantity of fluid seen. The primary aim of the FAST investigation was to detect intraperitoneal blood. Second, the aim of FAST was to detect pericardial fluid and blood. There was no attempt to evaluate visceral organ injury.

The FAST exam was performed in real time. There was no "second view" nor the radiologists give a follow-up report. CECT scans of abdomen were performed in all patients, Asteion spiral CT machine was used. Patients were classified into FAST +ve and FAST -ve. All the patients were operated and the findings of FAST and CECT correlated with peroperative findings. Data were tabulated and sensitivity of investigations analysed.

\section{RESULTS}

A total of 50 cases of blunt injury abdomen were included in this study. Of these cases, 44 cases (88\%) were males and 06 cases $(12 \%)$ were females. The range of age of the patients studied was from 16 years to 72 years and the mean age of the study group was 37.22 years.

The greatest distribution of cases was found in the $31-40$ years' age group with 16 cases (32\%) followed by $21-30$ years' age group with 14 cases (28\%) and 41 - 50 years' age group with 12 cases (24\%). Only 2 cases (4\%) were documented in the $<20$ years' age group and one case $(2 \%)$ in the $>60$ years' age group. Cumulatively, the age groups of 21 - 50 years represented the bulk of the study population with 42 cases $(84 \%)$.

\begin{tabular}{|c|c|c|c|c|}
\hline Age (in yrs.) & Male & Female & Total & \% \\
\hline$<20$ & 02 & 00 & 02 & 04 \\
\hline $21-30$ & 13 & 01 & 14 & 28 \\
\hline $31-40$ & 16 & 00 & 16 & 32 \\
\hline $41-50$ & 09 & 03 & 12 & 24 \\
\hline $51-60$ & 03 & 02 & 05 & 10 \\
\hline$>60$ & 01 & 00 & 01 & 02 \\
\hline Total & $\mathbf{4 4}$ & $\mathbf{0 6}$ & $\mathbf{5 0}$ & \\
\hline Table 1. Age and Sex Distribution of Study Population \\
\hline
\end{tabular}

In our study, Road traffic accidents were the predominant cause of blunt injury with 43 cases (86\%) being attributed to Road traffic accidents. Rest of the cases were due to fall from height (Figure 1).

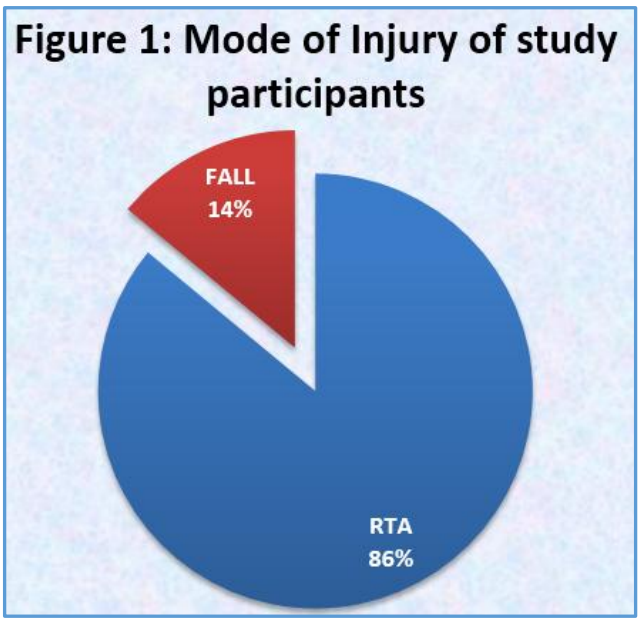

The findings of $\mathrm{x}$-ray chest/ abdomen erect showed air under diaphragm in $6 \%$, ground glass appearance in $20 \%$ and no abnormality in $74 \%$ of study participants (Figure 2 ).

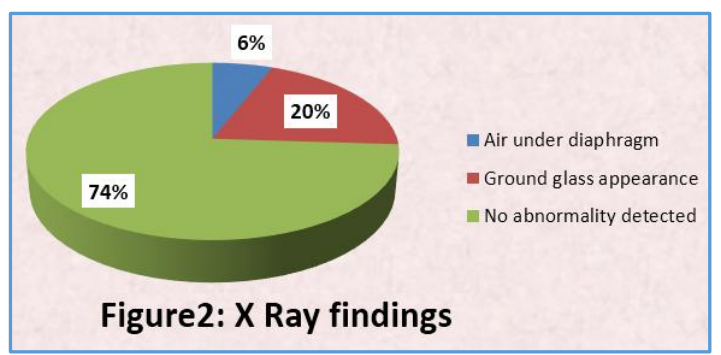

FAST (Focussed Abdominal Sonography in Trauma) was done in all 50 cases. FAST showed that the findings were positive in 49 patients and negative in one patient (Table 2). The positive findings were present in right hypochondrium in $30 \%$, left hypochondrium in $44 \%$ and Morrison pouch in $10 \%$ of patients (Table 3).

\begin{tabular}{|c|c|c|}
\hline Result & No. of Patients & Percentage \\
\hline Positive & 49 & $98 \%$ \\
\hline Negative & 1 & $2 \%$ \\
\hline \multicolumn{2}{|c|}{ Table 2. Results of FAST among Study Patients } \\
\hline
\end{tabular}




\begin{tabular}{|c|c|c|}
\hline Quadrants & No. of Patients & Percentage \\
\hline Morrison pouch & 5 & $10 \%$ \\
\hline Right hypochondrium & 15 & $30 \%$ \\
\hline Left hypochondrium & 22 & $44 \%$ \\
\hline Pelvis & 2 & $4 \%$ \\
\hline Combined & 5 & $10 \%$ \\
\hline \multicolumn{3}{|c|}{ Table 3. Findings in FAST Positive Patients } \\
\hline
\end{tabular}

CECT findings were positive in all 50 patients. CECT findings showed splenic injury in $40 \%$, liver injury in $32 \%$, multiple organ injury in $18 \%$ and other injuries in remaining patients (Figure 3).

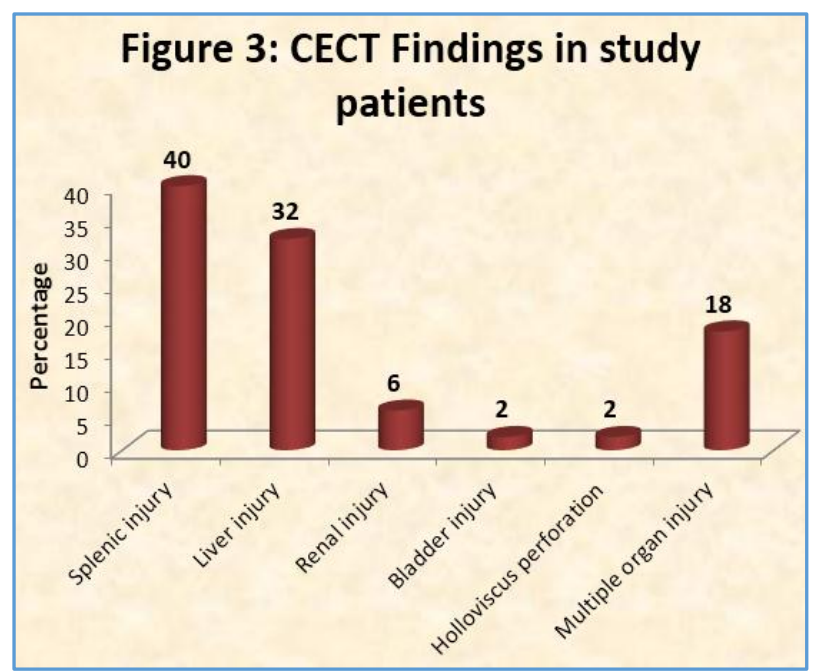

FAST and CECT findings were correlated with peroperative findings. Per-operative findings were positive in 50 patients. 49 patients were correctly identified by FAST (sensitivity 98\%) and CECT correctly identified all 50 patients (sensitivity 100\%).

\begin{tabular}{|c|c|c|c|}
\hline Investigation & $\begin{array}{c}\text { No. of Pts. } \\
\text { Positive }\end{array}$ & $\begin{array}{c}\text { No. of Pts. } \\
\text { Negative }\end{array}$ & Sensitivity \\
\hline FAST & 49 & 1 & $98 \%$ \\
\hline CECT & 50 & 0 & $100 \%$ \\
\hline \multicolumn{4}{|c|}{ Table 4. Comparison of FAST vs CECT } \\
\hline
\end{tabular}

Per-operative findings showed injury in spleen in 50\%, liver in $32 \%$, kidney in $16 \%$ and mesentery in $10 \%$ (Figure 4).

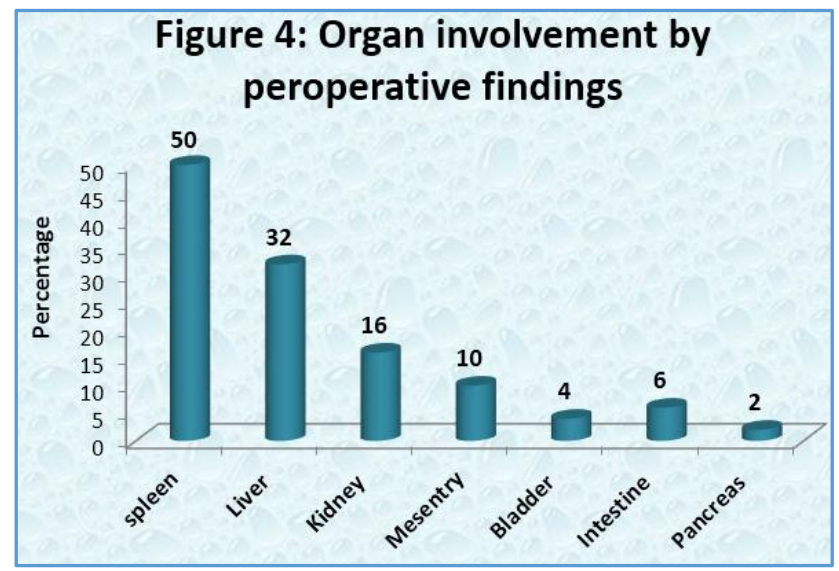

\section{DISCUSSION}

In the present study, 50 patients with blunt abdomen injury were studied. RTA is the most common mode of injury. FAST and CECT abdomen findings were compared with preoperative findings. The sensitivity of FAST was $98 \%$ and CECT was $100 \%$.

\begin{tabular}{|c|c|c|c|}
\hline Cause & Our Study & Davis et al $^{\mathbf{9}}$ & Khanna et al $^{10}$ \\
\hline $\begin{array}{c}\text { Road Traffic } \\
\text { Accident }\end{array}$ & $86 \%$ & $70 \%$ & $57 \%$ \\
\hline $\begin{array}{c}\text { Fall from } \\
\text { Height }\end{array}$ & $14 \%$ & $6 \%$ & $15 \%$ \\
\hline \multicolumn{3}{|c|}{ Table 5. Mode of Injury Comparison } \\
\hline
\end{tabular}

From the above table, it can be clearly seen that RTA is the most common mode of injury because of increased number of vehicles recently. The young people also give priority to speed rather than safety.

Plain erect x-ray of abdomen was done in all cases. Gas under diaphragm was found in 3 cases. In Davis et al study, abdominal x-ray was abnormal in $21 \%$ cases. In our study, it is $6 \%$ abnormal.

USG abdomen (Focussed Abdominal Sonography for Trauma) was done in all cases, out of which 47 cases had solid organ injury. Therefore, USG abdomen is more reliable in detecting solid organ injury and free fluid in the abdomen. Focussed Abdominal Sonography in Trauma (FAST) examination of pericardial, perihepatic, perisplenic and pelvic areas help in early detection of clinically important abdominal injury. FAST exam can be performed number of times and is an excellent tool in addition to physical examination.

CECT was done in all cases. It was positive in all cases. Contrast enhanced computed tomography (CECT) can provide trusted information on haemoperitoneum, grade of solid organ injuries, retroperitoneal organ injuries, most case of hollow viscus damage and ongoing bleed by means of radiographic blushing.

In liver injury, ultrasound (FAST) can reveal a break in the liver contour and disruption of normal hepatic architecture. Fluid that is presumed to be blood may be visualised around the liver and can be used as a screening procedure. CT scan can specifically identify an injury such as disruption of hepatic architecture and intrahepatic bleeding, subcapsular or intrahepatic haematoma and peri-hepatic blood can be seen. ${ }^{11}$ An infusion CT scan might reveal decreased density in an area of liver with compromised blood perfusion. The correlation of extent of injury by CT and that found at the time of operation may not be precise with instances of both over and under estimation. Despite this limitation, CT has clearly become the gold standard among the imaging modalities available for evaluation of hepatic trauma. In the era of selective non-operative therapy, knowledge of the nature of the hepatic injury is essential in the selection of the therapy. ${ }^{12}$

The incidence of pancreatic injury in severe abdominal trauma patients is about $3 \%-12 \%$ with blunt trauma contributing to about $1 / 3$ of these patients. The spectrum in pancreatic injuries is wide, ranging from simple contusion to fracture/ laceration to complete disruption. ${ }^{13}$ Injuries to the body are associated with blunt trauma to transverse colon. Injuries to tail of pancreas are associated with injury to the spleen. Associated organ injuries to pancreatic injuries are 
frequent in liver, stomach, vascular system, small bowel and colon, spleen, kidney, duodenum and biliary tract on descending order. ${ }^{14}$

Most patients with injuries to the retroperitoneal pancreas will have minimal clinical symptoms and signs when seen first after trauma; reason for this is the retroperitoneal location of the organ which masks the early development of peritonitis. ${ }^{15}$ Computed tomography may provide direct information about the location of pancreatic injuries as well as providing 3-dimensional picture of the wound. 16

The more common sign in patients with traumatic damage to the genitourinary path is haematuria. Radiographic evaluation has been recommended in every trauma patients who has haematuria. A kidney contusion is seen as an area of poor opacified renal parenchyma. Since a kidney laceration extends to the surface of the kidney, a subcapsular or perirenal haematoma will be present. ${ }^{17}$

Arterial injury can be found by CECT if they cause renal infarction or if active extravasation is demonstrated. The infarcted area of renal parenchyma does not have enhancement with intravenous contrast injection. However, a thin rim of enhanced kidney can be detected if the kidney capsular vessels remain uninjured. Venous damage could result in either vessel laceration or thrombosis. Avulsion of the ureteropelvic junction is easily diagnosed on CECT by the extravasation of excreted contrast dye. ${ }^{18}$ Also haematoma is invariably present and other abdominal injury such as liver and splenic lacerations are common.

Investigations are done with two main purposes. Firstly to evaluate and stage the injury, and secondly to see for the presence and functional status of kidney on the other side. Urinalysis and Ultrasonography along with isotope study are useful preliminary screening procedures. Intravenous urography (IVU) with high-dose infusion nephrotomography demarcates renal outlines with or without extravasation or renal laceration. ${ }^{19}$ Ultrasonogram is of value in detecting preexisting or developing hydronephrosis, urinoma and pararenal pseudohydronephrosis. It is indicated early in cases of non-functioning kidney (on IVU) to detect parenchymatous state and site of haematoma- intrarenal or prerenal.20

\section{Computed Tomography Scan}

Is preferred modality of investigation for blunt injury. It provides better information of lacerations if present and blood collection within Gerota's fascia. Other organs of abdomen are also defined simultaneously. Small areas of infarct, $1 \mathrm{~cm}$ are easily detected on CT scan. In contrast enhanced CT, renal infarcts are classically described as cortical rim sign. In intestinal injuries, $\mathrm{x}$-ray chest/ abdomen will show air under diaphragm. FAST shows free fluid in the peritoneum. CECT detects free fluid, air loculated anywhere in the peritoneal cavity.

CECT is the gold standard technique for evaluating of blunt injury patients, because it is highly sensitive and panoramic compared with FAST. FAST (USG) is widely available, largely used as the preferred screening tool in many trauma centres. FAST has been observed as a valuable primary imaging technique because it is rapid and noninvasive, and can be used on bedside of the patient and it was found not to interrupt resuscitation efforts. FAST (USG) is easily repeatable and less expensive.

FAST has proven to be reliable in evaluating the presence of free intraperitoneal fluid. A literature reveals that FAST (USG) is an excellent tool for visualisation of haemoperitoneum with a sensitivity of $63 \%-99 \%$. The main limitation is its poor ability to depict parenchymal lesions.

The value of FAST in revealing an organ injury varies greatly according to the lesion located. In the detection of spleen injuries, the sensitivity ranges from $27 \%$ to $68.6 \%$. The bigger size of the liver and easier approach implies the higher sensitivity of FAST for lesion of liver, ranging from $51 \%$ to $87.5 \%$ in various studies. ${ }^{21}$ For renal or suprarenal injuries, however, FAST has a low sensitivity (25\% - 40\%). FAST is found to be a diagnostic tool initially to detect intraabdominal fluid in abdominal trauma. With proper training and understanding, the limitations of ultrasound and optimisation of the results of FAST is obtained.

In haemodynamically stable patients, the diagnostic modality of choice is CECT with intravenous contrast. It is found to be of use in detecting free air and intraperitoneal fluid, grade the extent of solid viscera injury, detect retroperitoneal organ injury and helps in the decision for a conservative management. Helical CECT is done rapidly which decreases the time the patient stays in the CECT scan room. Furthermore, CECT improves the sagittal and coronal reconstruction views which are of use for detecting a ruptured diaphragm.

Blunt injury abdomen with solid organ injury forms considerable load of patients in our society. Most common age group involved is 31 - 40 years. Predominantly, males are affected in large proportions. Road traffic accident forms the most common mode of injury. So efforts should be made to bring road traffic regulations into strict action and traffic norms to be regulated. Well established trauma care centres should be established at every Taluk hospital. Measures for early transport of the patients from the accident site to the trauma centres should be undertaken.

Clinical presentation is varied, sometimes confusing. Erect abdomen x-ray is a useful investigation to identify associated hollow viscus injury. This study observed FAST as adjunct to the initial evaluation of blunt injury patients. Those with a +ve FAST are at risk for significant abdominal bleeding that is critical and likely to need laparotomy sooner. Resource might then be mobilised appropriately and injury care hopefully revolutionised.

Therefore, FAST is found useful in the early decision making whether the patient needs immediate laparotomy to control the bleeding. Those with a -ve FAST are not at significant risk for bleeding and can be imaged by CECT in a less urgent manner. A particular minority of patients cannot be evaluated by FAST, and in those patients, other clinical investigations must be followed to decide treatment.

Additionally, FAST performed by the surgeon, as is so in our experience should only be used as a screening mode for bleeding and might not supplement a CECT scan as a definitive imaging mode for intra-abdominal trauma. With the advent of high resolution ultrasonography (FAST) DPL and FQA investigations are becoming less opted. CECT forms the core investigation of choice in dealing with blunt injury abdomen patients. 


\section{CONCLUSION}

FAST can be done in all blunt injury abdomen patients and the need for laparotomy assessed, as it detects solid organ injuries with sensitivity reaching $98 \%$ from this study. If the patient is stable, then further imaging by CECT can be done.

\section{REFERENCES}

[1] Thal ER. Abdominal-trauma, North American Surgical clinics. WB Saunders Company 1990;70(3):357-69.

[2] Blumgart LH, Fong Y. Surgery of the liver and biliary tract. Vol 1. 3 $3^{\text {rd }}$ edn. WB Saunders 2000: p. 1277-318.

[3] Feliciano DV. Diagnostic modalities in abdominal trauma. Peritoneal lavage, Ultrasonography, computed tomography scanning and arteriography. Surgical Clinics of North America 1991;71(2):241-56.

[4] Scalea TM, Rodriguez A, Chiu WC, et al. Focussed Assessment with Sonography for Trauma (FAST): results from an international consensus conference. J Trauma 1999;46(3):466-72.

[5] McAnena OJ, Moore EE, Marx JA. Initial evaluation of the patient with blunt abdominal trauma. Surgi Clin North Am 1990;70(3):495-515.

[6] Schwab CW. Selection of non-operative management candidates. World J Surg 2001;25(11):1389-92.

[7] Stawicki-SP. Trends of non-operative management of traumatic injuries: a synopsis. OPUS 12 Scientist 2007;1(1):19-35.

[8] Gupta S, Talwar S, Sharma RK, et al. Blunt Trauma Abdomen: a study of 63 cases. Indian J Med Sci 1996;50(8):272-6.

[9] Davis JJ, Cohn I, Nance FC. Diagnosis and management of blunt abdominal trauma. Ann Surg 1976;183(6):672-8.

[10] Khanna R, Khanna S, Singh P. Spectrum of blunt abdominal trauma in Varanasi. Quart J 1999;35(1\&2):25-8.
[11] Carrillo EH, Wohltmann C, Richardson JD, et al. Evolution of the treatment of complex blunt liver injuries. Current Problems in Surgery 2001;38(1):960.

[12] Trunkey DD, Shires GT, McClelland R. Management of liver trauma in 811 consecutive patients. Ann Surg 1974;179(5):721-8.

[13] Knudson MM, Maull KI. Non-operative management of solid organ injuries. Past, present and future. Surg Clin North Am 1999;79(6):1357-71.

[14] Goins WA, Rodriguez A, Lewis J, et al. Retro-peritoneal hematoma after blunt trauma. Surg Gynecol Obstet 1992;174(4):281-90.

[15] Carlin AM, Tyburski JG, Wilson RF, et al. Factors affecting the out-come of patients with splenic trauma. American Surg 2002;68(3):232-9.

[16] Velmahos GC, Toutouzas KG, Radin R, et al. Nonoperative treatment of blunt injury to the solid abdominal organs: a prospective study. Arch Surg 2003;138(8):844-51.

[17] Corriere JN, Sandler CM. Management of ruptured bladder: seven years of experience with 111 cases. J Trauma 1986;26(9):830-3.

[18] McConnell DB, Trunkey DD. Non-operative management of abdominal trauma. Surg Clin North Am 1990;70(3):677-88.

[19] Jurkovich GJ, Carrico CJ. Trauma: management of the acutely injured Patient. In: Sabiston DC. edr. Text-book Surgery. Noida: Thomson Press (India) Ltd., 1997: p. 297-336.

[20] Gupta, Lall R. Recent advances in surgery. New Delhi: Jaypee Brothers 1998;6:140-8.

[21] Cox EF. Blunt abdominal trauma. A 5-year analysis of 870 patients requiring celiotomy. Ann Surg 1984;199(4):467-74. 